\title{
loT meets logistics - \\ Development of a mobile condition monitoring system
}

\author{
Michael Heider ${ }^{1}$, Dr. André Rother ${ }^{1}$, Dr. Paul Hanesch ${ }^{1}$ \\ 1 WIKA Alexander Wiegand SE \& Co. KG, Klingenberg am Main, Germany, \\ Michael.Heider@wika.com
}

\begin{abstract}
:
It is common to determine the position of goods via GPS in the delivery process, however the conditions of specific commodities are insufficiently tracked. Advances in communication technologies combined with development of low energy sensors offer several new possibilities in continuous online monitoring. To overcome the aforementioned problems, we investigated the delivery process in the field of roadworks. In this field there are numerous opportunities into which loT can be implemented. For that reason we developed an innovative monitoring system concept for the supply chain of tarmac. The prototype enables the wireless transmission of logged temperature data which are coming from several sensors placed at certain positions on the trailer.
\end{abstract}

Key words: Condition Monitoring, Wireless, Cloud, Temperature, Data logging

\section{Introduction}

Nowadays it is commonplace to determine the current whereabouts of a delivery via several checkpoints in different locations throughout the delivery process, e.g. the transfer of a package from the sender to the postman. The condition of the delivery can also be ascertained via an indicator upon delivery, such as an acceleration tag [1] that changes color if a specified maximum value has been exceeded.

However, if a failure occurs during transport, the recipient has no information about the influences, for example, where and when the environmental conditions exceeded their specified limits. Growing progress in the digitalization referring to the internet of things (loT) has led to a sensitization of markets, which in the past, were not exposed to such technologies [2].

We choose to investigate the field of roadworks because of the mentioned shortcomings in monitoring of the supply chain. This offers us the opportunity to demonstrate the integration of loT into that field. The one we chose to investigate further, being condition monitoring of tarmac during transportation. If the temperature of the tarmac decreases below a certain level, the road workers cannot guarantee that the new road will meet the quality requirements. Crucial, is being able to determine, in case of a problem, who in the delivery chain is responsible.
If the quality requirement of the tarmac doesn't matched the standards, expensive repair work could be the consequence. Currently no solution exists for live monitoring of the temperature of the tarmac during transportation.

\section{Concept}

Our approach is the development and evaluation of a concept for online monitoring of the delivery process of tarmac. In Figure 1 the basic prototype is shown. The functionalities and components of that prototype are described below.

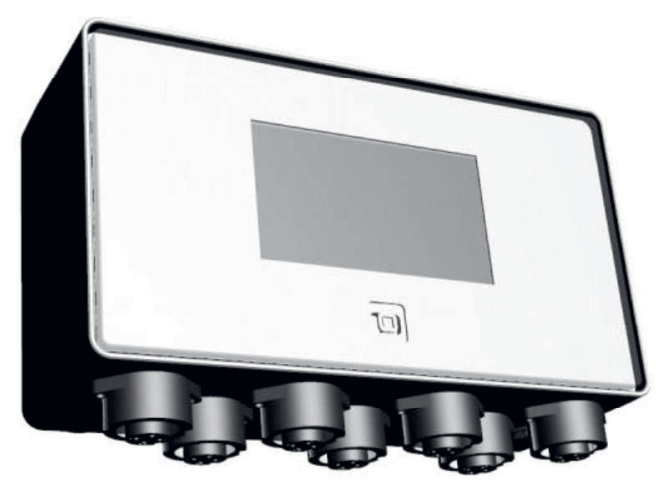

Fig. 1: First prototype of a tarmac monitoring system

In order to supply the prototype, we integrated the monitoring device into the electrical system of the truck delivering the tarmac. For that reason all of the electronic components are designed to operate with $24 \mathrm{~V}$ and below. First of all, there is an automotive protection circuit to 
cover the electronics of the monitoring system and to start the system when the ignition is activated.

The main portion of the electronics is the controller unit with an integrated GSM module. The controller is responsible for the signal processing, as well as the wireless transmission via cellular connection. In addition to the GSM module, there is also a Bluetooth and a GPS module connected to the controller. Up to 5 temperature sensors can be connected via cable to the device and be digitalized using highly accurate analog digital converter, which is designed to work even under harsh environmental conditions. Moreover, the temperature values can be logged in an internal extendable memory for the entire duration of the delivery.

This offers different possibilities for the further processing of the data. When the truck reaches its destination, the responsible worker has the advantage to reconstruct the temperature curve of the tarmac during the delivering process. So even before the deployment of the tarmac it is clear where and when the temperature decreased below the minimum temperature in case of a failure.

An internal Bluetooth interface allows the worker to send the data to an handheld Bluetooth printer. This enables one to print out a protocol of the reported data via the handheld printer. The cellular communication unit allows a continuous data transfer of the logged data to a specific server. The latter offers a graphical user interface (GUI) with different functionalities including live monitoring of the temperatures during the delivery.

The prototype was tested under real conditions during several tarmac deliveries and the results are discussed below.

\section{Results}

The first important point is the complexity of the installation of the monitoring system. The prototype should be easy to put into operation. Because of that, a step by step instruction was created describing how to place and connect the temperature sensors. Figure 2 depicts the position of the multi-array of temperature sensors which are necessary to ensure reliable condition monitoring of the tarmac in the trailer. After installation is completed, the monitoring system is ready to use without any further IT support.

As described in the concept, the logged temperature values are transferred to a cloud server which offers several options to process the received data. The basic function is the storage of the temperature values in a database, ensuring that all deliveries from previous years can be evaluated. Another significant point is the visualization of the received data. The GUI enables one to display

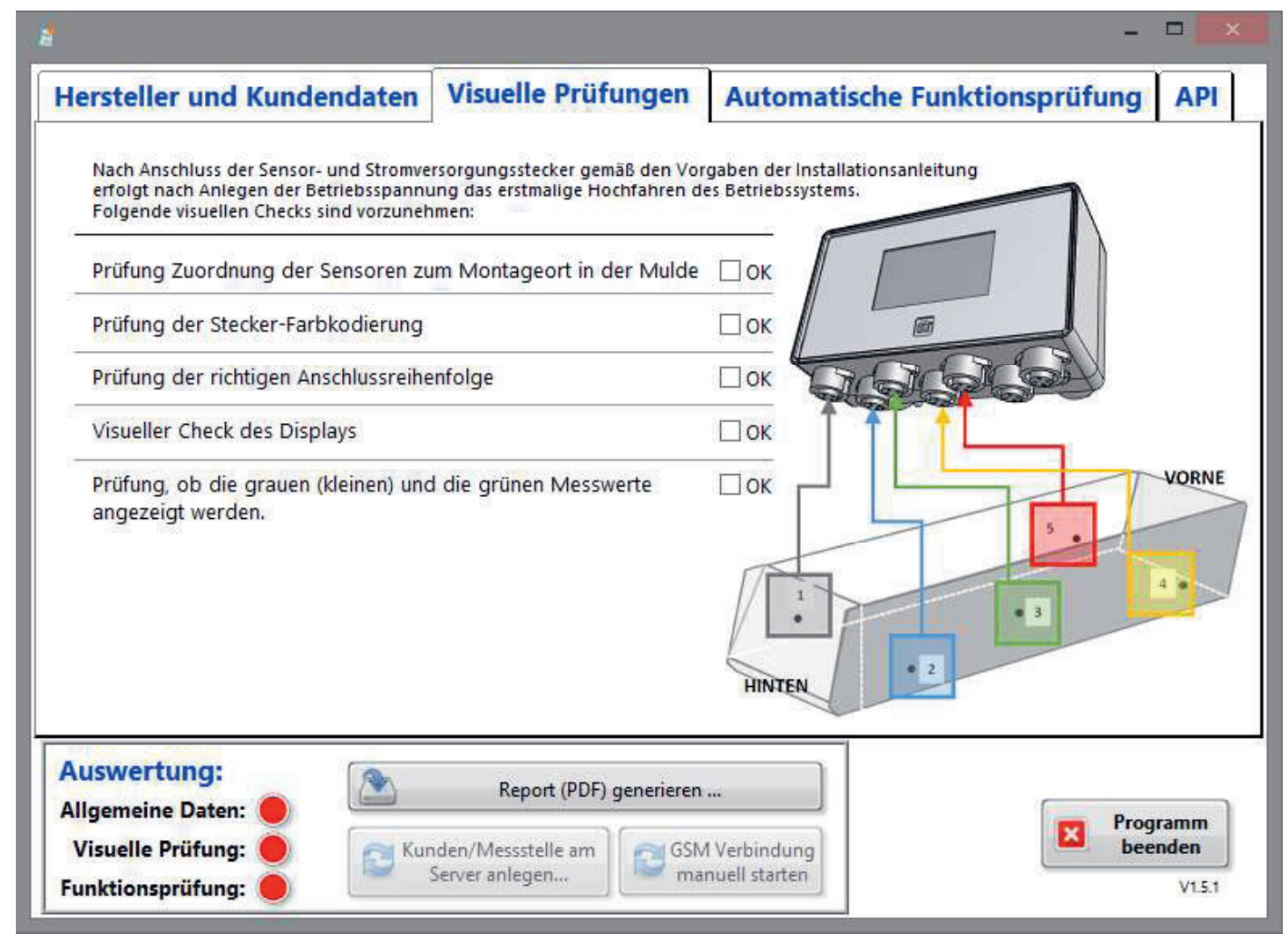

Fig. 2: Position of the temperature sensors in the tarmac trailer 
the temperature course using different graphs. One example of the visualization of a delivery process is shown in figure 3 .

\section{Further Steps}

Now that the basis prototype is running, there are some points that may be optimized or added to the existing system.

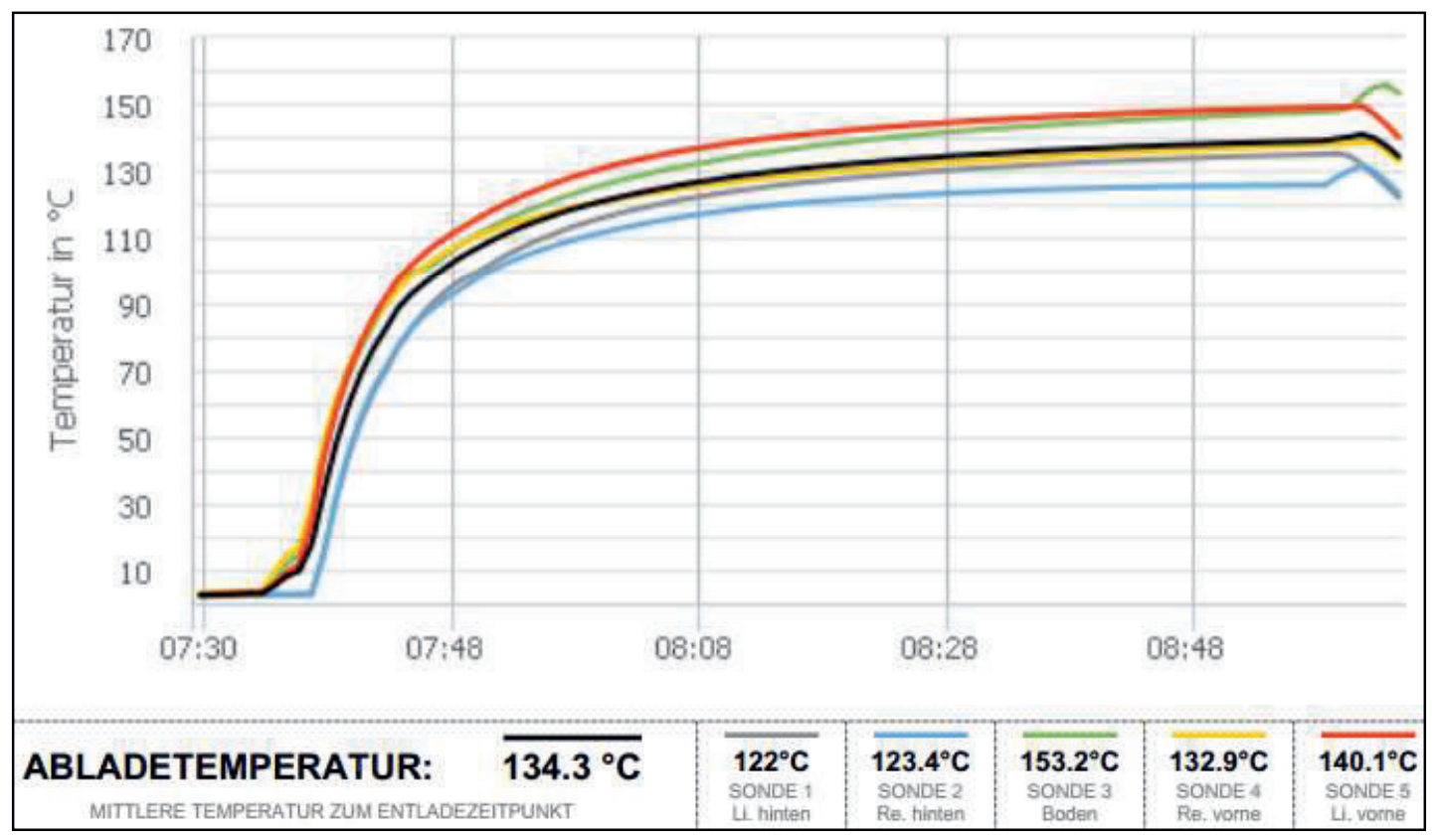

Fig. 3: Temperature history of a whole tarmac delivery process provided by the GUI of the server

Besides the storage and visualization of the temperatures there exists the option to set alarms. This means that an automated message via e-mail will be sent if the temperature drops below a certain value. In that case the delivery can be stopped even before the trailer arrives at the construction site.

During the real time tests, the same temperature sensors which were placed in the trailer and also in the tarmac were used as references in order to qualify the monitoring system. During these tests, a challenge regarding the temperature measurement of the tarmac arose. When the hot tarmac is loaded in the trailer the temperature of the tarmac which is in contact with the side drops by approx. $30 \mathrm{~K}$. These colder layers insulate the inner tarmac from cooling too rapidly. The challenge in this case was being able to determine the correct temperature of the inner tarmac, while only being able to measure the temperature of the outer layer.

For that reason we had to develop an algorithm to calculate the correct temperature inside of the trailer. All in all, the ambient temperature and the type of trailer define the conversion factor between the inner and outer temperature of the tarmac. The calculations are performed by the control unit at the trailer and the corrected values are sent to the cloud server.
One possible modification could be the connection between the temperature sensors and the monitoring device. The reason is that the wiring of the sensors is error-prone due to the harsh environmental conditions they are exposed to. The solution to that problem could be to replace the wired connection with a wireless connection such as Bluetooth or Wi-Fi.

Another point which could have a substantial benefit is the connection of the trucks control unit to the monitoring system. This would enable the transfer of other information, for example data from the engine or status of wear parts to the cloud, providing the transportation company with not only the status of deliveries, but information about the condition of the truck itself.

\section{References}

[1] A. Davies, Handbook of Condition Monitoring, Techniques and Methodology, SpringerScience+Business Media, 1998

[2] O. Vermesan, P. Friess, Internet of Things Global Technological and Societal Trends, River Publishers, 2011 